\title{
Biodegradabilidade e Propriedades Mecânicas de Novas Misturas Poliméricas
}

\author{
Derval S. Rosa, Bruno L. M. Franco, Maria Regina Calil \\ Laboratório de Polímeros Biodegradáveis e Soluções Ambientais do Centro de Ciências Exatas e \\ Tecnológicas da Universidade São Francisco
}

Resumo: O plástico tem sido cada vez mais usado em nosso cotidiano em diversos tipos de produtos: garrafas, embalagens, automóveis, etc. Diversas alternativas têm sido buscadas para minimizar o impacto ambiental causado pelos polímeros convencionais. Uma importante alternativa é o uso de polímeros biodegradáveis, que podem ser degradados pela ação de microorganismos, tais como bactérias, fungos ou algas. As aplicações tecnológicas de polímeros biodegradáveis normalmente requerem melhorias nas suas propriedades mecânicas. Neste contexto, novas misturas poliméricas vêm sendo obtidas para aplicações inovadoras e mais baratas. Neste trabalho, será apresentada a metodologia de preparação de novas misturas poliméricas, contendo diferentes teores de amido, com Policaprolactona (PCL), Polihidróxibutirato (PHB) e um copolímero Poli(hidróxibutirato-co-valerato) (PHBV). A resistência à tração das blendas com 50\% em massa de amido é $35 \%$ e $60 \%$ menor do que a dos polímeros PCL e PHBV puros, respectivamente. Quando expostas a microorganismos em lodo ativado, as misturas de PCL ou PHBV com maiores dosagens de amido apresentam maiores taxas de degradação.

Palavras-chave: Misturas, polihidróxibutirato, policaprolactona, poli(hidróxibutirato-co-valerato), amido.

\section{Biodegradability and Mechanical Properties of Polymeric Mixtures}

\begin{abstract}
Plastics are finding increasing application in our daily life, in products such as bottles and automobiles, for packing, etc. Several possibilities have been considered to minimize the environmental impact caused by the use of conventional polymers. An important alternative is the biodegradable polymer, which can be degraded through the action of naturally occurring microorganisms, such as bacteria, fungi or algae. Technological applications of biodegradable polymers usually require improvements on their mechanical properties. In this context, novel polymer blends have been obtained for inexpensive applications. In the present work, the methodology of preparation of new polymer blends is described. The blends contain different quantities of starch, with Poly (e-caprolactone)-(PCL), Poly (ß-hydroxybutyrate)-(PHB) and Poly (ß-hydroxybutyrate-cob-hydroxyvalerate)-(PHBV). The resistance to the tensile strength of the blends with $50 \%$ in mass of starch was $35 \%$ and $60 \%$ lower than for the pure PCL and PHBV, respectively. Upon exposure to microorganisms in activated mud, the mixtures of PCL or PHBV with larger amounts of starch display higher rates of degradation.
\end{abstract}

Keyword: Mixtures, poly ( $\beta$-hydroxybutyrate), poly (e-caprolactone), poly ( $\beta$-hydroxybutyrate-co-bhydroxyvalerate) and starch..

\section{Introdução}

Os produtos produzidos com os polímeros sintéticos convencionais são considerados inertes ao ataque imediato de microorganismos. Essa propriedade faz com que esses materiais apresentem um tempo longo de vida útil e, consequentemente, provocam sérios problemas ambientais visto que, após o seu descarte, demoram em média 100 anos para se decomporem totalmente ${ }^{(1-3)}$, aumentando assim a cada

Autor para correspondência: Derval dos Santos Rosa, Laboratório de Polímeros Biodegradáveis e Soluções Ambientais do Centro de Ciências Exatas e Tecnológicas da Universidade São Francisco, Rua Alexandre Rodrigues Barbosa, 45, Centro, CEP: 13.251-900, ITATIBA, -SP, Telefone (0xx 11) 4534-8046 /4534-8126.E-mail:derval@saofrancisco.edu.br. 
dia, a quantidade de lixo plástico descartado no meio ambiente ${ }^{[4]}$.

Como uma solução para este problema, surgem os polímeros biodegradáveis que quando entram em contato com diversos tipos de microrganismos se degradam rapidamente ${ }^{[1]}$.

Nos últimos anos tem havido um interesse crescente, em nível mundial, em relação aos polímeros biodegradáveis, principalmente quando se considera o desenvolvimento de novos produtos que provoquem menor impacto ambiental. No entanto, devido o seu alto custo quando comparados com polímeros convencionais, para a maioria das empresas, a utilização de tais polímeros ainda é inviável. Como exemplo, pode-se citar o polietileno de baixa densidade (PEBD), utilizado em muitos produtos do nosso cotidiano como em embalagens, entre outros artefatos, cujo preço gira em torno de 1 dolar o quilo, enquanto que o quilo de um polímero biodegradável custa, em média, 5 a 8 dólares ${ }^{[4]}$.

Como uma solução para a questão do alto custo desses materiais, emerge a necessidade de desenvolvimento de misturas poliméricas biodegradáveis que consistam numa mistura física de dois ou mais tipos de materiais poliméricos ou copolímeros que não são ligados por ligações covalentes ${ }^{[5]}$.

$O$ recurso de se utilizar um aditivo natural degradável para as formulações de plásticos biodegradáveis não é nova. Aditivos naturais tais como o amido, a celulose, a lignina, entre outros, são usados em formulações de plásticos convencionais $^{[6-8]}$.

O amido é uma matéria prima abundante que permite o desenvolvimento de produtos recicláveis. Quando biodegradado ou incinerado este material libera $\mathrm{CO}_{2}$ para a atmosfera. Estas características do amido tem despertado um interesse acentuado pelo mercado de polímeros convencionais através da sua incorporação em misturas poliméricas ${ }^{[6-8]}$.

Neste trabalho foram preparadas misturas poliméricas usando o amido derivado do milho, como aditivo, em mistura com os polímeros biodegradáveis PCL, PHB, e PHBV, com o objetivo de se obter novos materiais que apresentassem uma maior velocidade de degradação, ou seja, a obtenção de materiais que após o seu descarte teria consequências menos danosa ao ambiente. Além disso, esses polímeros teriam um custo mais baixo, uma vez que o amido é um material de baixo custo (valor estimado de U\$ $0,50 / \mathrm{kg}$ ).

\section{Experimental}

\section{Materiais}

Para a preparação das misturas, foram utilizados os seguintes polímeros biodegradáveis:

a-) Polihidroxibutirato - (PHB) da safra 98/99 Lote 62, da Copersucar (Cooperativa de Produtores de Cana, Açúcar e Álcool do Estado de São Paulo), com massa molar média de $80.000 \mathrm{~g} / \mathrm{mol}$ e $0,19 \%$, em massa, de nitrogênio e $0,66 \%$ em massa, de cinza.

b-) Copolímero poli(hidroxibutirato-co-valerato) (PHBV) da safra de 98/99, lote 54 da Copersucar ( Cooperativa de Produtores de Cana de Açúcar do Estado de São Paulo), com massa molar média de $150.000 \mathrm{~g} / \mathrm{mol}$, e teor de $5,5 \%$, em massa, de valerato, $0,09 \%$, em massa, de nitrogênio e $0,27 \%$, em massa, de cinza.

c-) Policaprolactona - (PCL) - polímero do tipo P-767 da Union Carbide Química Ltda. ${ }^{[9]}$, com índice de fluidez de 1,9 $\pm 0,3 \mathrm{~g} / 10 \mathrm{~min}$ (ASTMD-1238), densidade de $1,14 \mathrm{~g} / \mathrm{cm}^{3}$ e massa molar de $80.000 \mathrm{~g} / \mathrm{mol}$.

d-) Amido de milho do tipo Amidex 3001, contendo $27 \%$, em massa, de amilose e $73 \%$, em massa, de amilopectina, com massa molar média de $486.000 \mathrm{~g} / \mathrm{mol}$, fornecido pela Corn Products Brasil.

\section{Preparação das Misturas}

As misturas foram preparadas utilizando uma extrusora Werner Pfleiderer com dupla rosca de cisalhamento médio. As formulações desenvolvidas foram: a-) Formulação contendo $75 \%$ de PCL e $25 \%$ de amido preparadas com temperaturas entre 60 a $100^{\circ} \mathrm{C}$; b-) Formulação contendo 50\% de PCL e 50\% de amido preparadas com temperaturas entre 60 a $100^{\circ} \mathrm{C}$; c-) Formulação contendo $75 \%$ de $\mathrm{PHBv}$ e $25 \%$ de amido preparadas com temperaturas entre 160 a $180^{\circ} \mathrm{C}$; d-) Formulação contendo 50\% de PHBv e 50\% de amido preparadas com temperaturas entre 170 a $230^{\circ} \mathrm{C}$; e-) Formulação contendo $75 \%$ de PHB e $25 \%$ de amido preparadas com temperaturas entre 150 a $180^{\circ} \mathrm{C}$ e f-) Formulação contendo 50\% de PHB e 50\% de amido preparadas com temperaturas entre 170 a $230^{\circ} \mathrm{C}$.

\section{Preparação dos corpos-de-prova por Injeção}

Os corpos-de-prova, segundo Norma ISO R179, foram preparados pelo processo de injeção utilizan- 
do uma máquina injetora da marca PIC, Modelo PIC 62. Para o PCL a velocidade da rosca foi de 100rpm com curso de dosagem de $65 \mathrm{~mm}$, abertura de $150 \mathrm{~mm}$ e temperaturas nas diferentes zonas de $60-70^{\circ} \mathrm{C}$. No caso do $\mathrm{PHB} / \mathrm{V}$ e $\mathrm{PHB}$ a velocidade da rosca de $250 \mathrm{rpm}$, com cursos de dosagem de $50 \mathrm{~mm}$ e de abertura de $140 \mathrm{~mm}$, sendo que o curso de abertura no PHB foi de $150 \mathrm{~mm}$ e as temperaturas das diferentes zonas variaram de $135-170^{\circ} \mathrm{C}$.

A Formulação 4, contendo PHB com 50\% de amido, não se conseguiu determinar condições de processamento para injetar os corpos-de-prova perfeitos, provavelmente pela imiscibilidade dos materiais.

\section{Ensaios Mecânicos}

Os ensaios de tração foram realizados de acordo com a Norma ASTM-D-638 em uma Máquina Universal Marca EMIC Modelo DL-2000, seguindo as seguintes condições: temperatura de $25{ }^{\circ} \mathrm{C}$; umidade relativa de $50 \%$; velocidade da garra de $50,0 \mathrm{~mm} /$ min e distância entre garras de $115 \mathrm{~mm}$.

Nesse ensaio verificou-se as propriedades de resistência à tração $\left(\sigma_{\max }\right)$, alongamento na ruptura $(\varepsilon)$ e módulo de elasticidade (E).

\section{Microscopia Eletrônica de Varredura (MEV)}

As superfícies para análise por MEV foram obtidas através da retirada de um pedaço do corpo-deprova original dos ensaios mecânicos, as quais foram metalizados em um metalizador SCO-040, Marca Balzers, utilizando "sputtering" sob as seguintes con- dições: material de metalização, ouro; corrente elétrica, 33 mA; pressão, 0,05 mbar de argônio; tensão, $312 \mathrm{~V}$ e tempo de deposição $50 \mathrm{~s}$ em gás argônio.

As amostras foram analisadas em um microscópio eletrônico de varredura (MEV) Jeol JSM-35CF, em diferentes ampliações, nas seguintes condições: tensão de $15 \mathrm{kV}$ e corrente de emissão $0,2 \times 10^{-10} \mathrm{~A}$, após fratura criogênica utilizando nitrogênio líquido.

\section{Método de Liberação de $\mathrm{CO}_{2}{ }^{[10]}$}

A metodologia utilizada para determinar a quantidade de dióxido de carbono liberada pelos polímeros, quando exposto em água de esgoto e lodo ativado em condições laboratoriais, foi de acordo com a Norma ASTM 5209-92.

A Figura 1 ilustra como foi montado o sistema utilizado para captar e complexar o $\mathrm{CO}_{2}$ liberado pelo polímero. Nesse sistema, o polímero é adicionado no recipiente $\mathrm{B}$ (reator), que contém o lodo ativado. Para se evitar a entrada de $\mathrm{CO}_{2}$ no reator, é adicionado $\mathrm{Ba}(\mathrm{OH})_{2}$ no recipiente $\mathrm{A}$, garantindo assim que apenas o $\mathrm{O}_{2}$ entre no recipiente $\mathrm{B}$. $\mathrm{O} \mathrm{CO}_{2}$ gerado no reator reage com o hidróxido de bário formando $\mathrm{BaCO}_{3}$ e a quantidade de dióxido de carbono é determinada por retrotitulação com $\mathrm{HCl}$ do recipiente $\mathrm{C}$.

\section{Resultados e Discussão}

\section{Propriedades Mecânicas}

As propriedades mecânicas das formulações e dos polímeros puros foram caracterizadas de acordo com

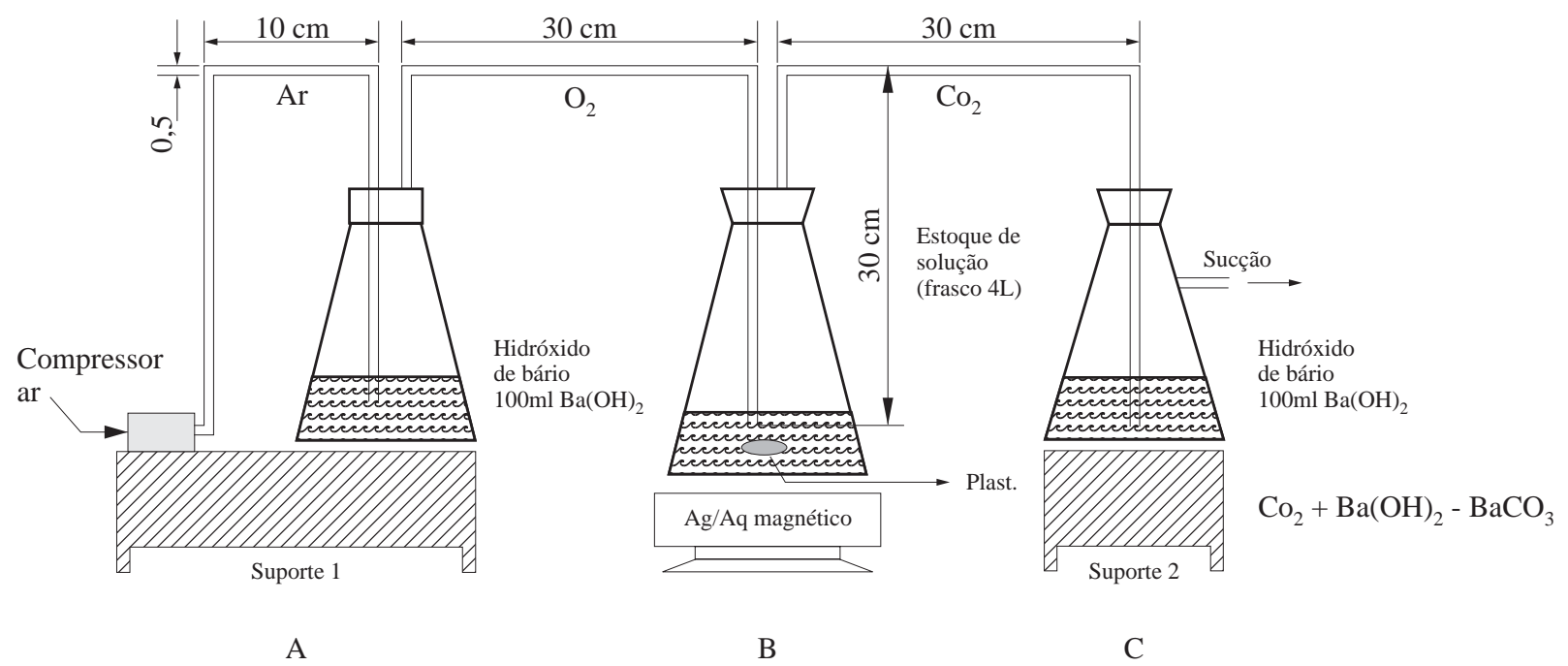

Figura 1. Esquema do equipamento utilizado para captar e complexar o dióxido de carbono. 
Tabela 1. Resultados médios dos ensaios mecânicos das formulações e polímeros puros e seus respectivos desvios padrão.

\begin{tabular}{cccc}
\hline Formulação & $\begin{array}{c}\sigma_{\text {maxima }} \\
(\mathbf{M P a})\end{array}$ & $\begin{array}{c}\boldsymbol{\varepsilon} \\
(\boldsymbol{\%})\end{array}$ & $\begin{array}{c}\mathbf{E} \\
(\mathbf{M P a})\end{array}$ \\
\hline PCL (puro) & $16,9 \pm 0,5$ & $393,0 \pm 29,3$ & $432,9 \pm 47,4$ \\
PCL 25\% amido & $14,5 \pm 0,2$ & $302,9 \pm 67,7$ & $306,0 \pm 15,9$ \\
PCL 50\% amido & $11,0 \pm 0,7$ & $3,5 \pm 0,1$ & $520,9 \pm 22,5$ \\
PHB (puro) & $28,6 \pm 2,7$ & $0,7 \pm 0,1$ & $5633,7 \pm 948,8$ \\
PHB 25\% amido & $9,3 \pm 1,3$ & $1,4 \pm 0,2$ & $940,0 \pm 126,8$ \\
PHBV (puro) & $34,2 \pm 1,0$ & $2,6 \pm 0,9$ & $3940,6 \pm 323,3$ \\
PHBV 25\%amido & $18,9 \pm 0,5$ & $3,3 \pm 0,4$ & $1191,8 \pm 61,2$ \\
PHBV 50\% amido & $13,8 \pm 0,5$ & $3,6 \pm 0,2$ & $924,4 \pm 72,8$ \\
\hline
\end{tabular}

o descrito nos Ensaios Mecânicos e os resultados estão apresentados na Tabela 1.

As curvas típicas de tensão vs. deformação obtidas durante os ensaios mecânicos para as formulações contendo PCL, PHB e PHBV, são apresentadas nas Figuras 2, 3 e 4, respectivamente.

A incorporação do amido, de uma maneira geral, reduziu os valores da tensão de tração na ruptura. Para o PCL ocorreu uma redução de $14 \%$, para o PHB de $69 \%$ e PHBV de $44 \%$ na propriedade com a incorporação de $25 \%$, em massa de amido. Para as misturas com $50 \%$, em massa o amido provocou uma redução mais severa em $35 \%$, no caso do PCL e $60 \%$ no do PHBV quando comparados com os valores da propriedade dos materiais puros.

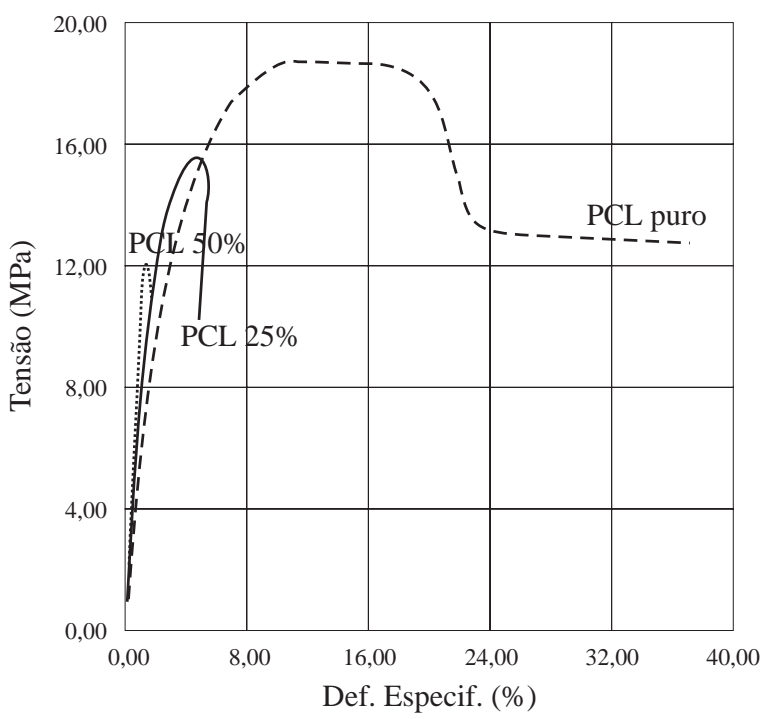

Figura 2. Curva tensão-deformação do polímero PCL e suas respectivas fomulações.

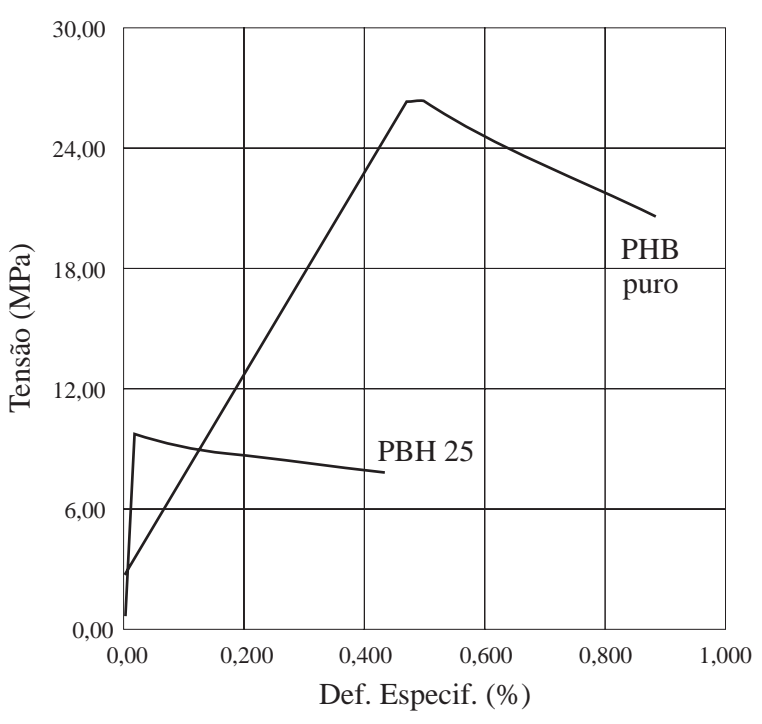

Figura 3. Curva tensão-deformação do polímero PHB e suas respectivas formulações.

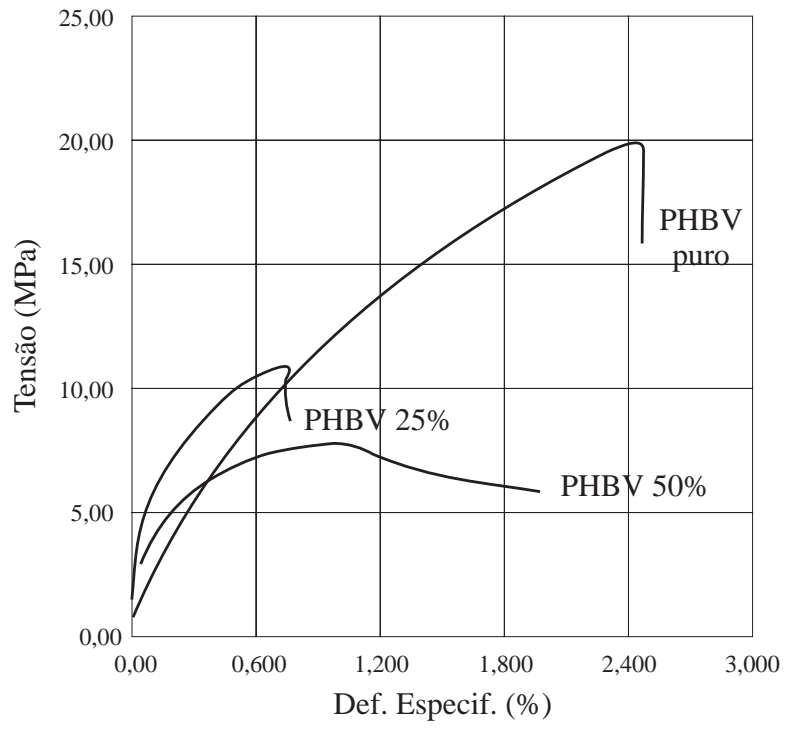

Figura 4. Curva tensão-deformação do polímero PHBV e suas respectivas formulações.

Não foi conseguida a preparação dos corpos-deprova dos ensaios mecânicos para a mistura de PHB com $50 \%$ de amido, provavelmente porque neste teor de amido ocorre uma forte incompatibilidade entre este material e o PHB.

Uma justificativa para a perda da propriedade mecânica é, provavelmente, pela pouca miscibilidade do amido com os polímeros estudados. Isto pode ser melhor explicado pelo fato do amido utilizado possuir uma cadeia molecular de tamanho grande (massa molar média $486.000 \mathrm{~g} / \mathrm{mol}$ ) e uma estrutura ramificada com a presença de anéis benzeno. Possivelmente a inclusão do amido provocou uma diminuição nas interações en- 
tre as cadeias dos polímeros, que são da família éster, e que apresenta grande quantidade de ligação $\mathrm{C}=\mathrm{O}$ que provoca grandes forças intermoleculares.

Um comportamento similar foi observado para o alongamento na ruptura e no módulo de elasticidade, exceto para a mistura de PCL contendo 50\%, em massa, de amido nesta última propriedade.

Na curva típica tensão $v s$. deformação foi observado um menor escoamento com adição de amido ao PCL, enquanto que para PHB e PHBV houve um aumento desta propriedade nas formulações contendo amido.

\section{Microscopia Eletrônica de Varredura}

As Figuras 5, 6 e 7 apresentam as fotomicrografias das amostras puras e as suas respectivas formulações.

Através das fotomicrografias de superfície das amostras foi possível evidenciar maior compatibilidade entre o PCL e o amido o que também pode ser evidenciado através dos resultados das propriedades mecânicas, onde é observado uma menor redução desta propriedade quando comparado com os outros polímeros puros estudados. Uma explicação para este fato é que na preparação das misturas contendo PCL, a faixa de temperatura utilizada foi de $97^{\circ} \mathrm{C}$. Nessa temperatura não ocorre a gelatinização do amido, ou seja, a abertura dos grãos, o que faz com que este atue apenas como carga na mistura e não diminuindo as interações entre as cadeias do

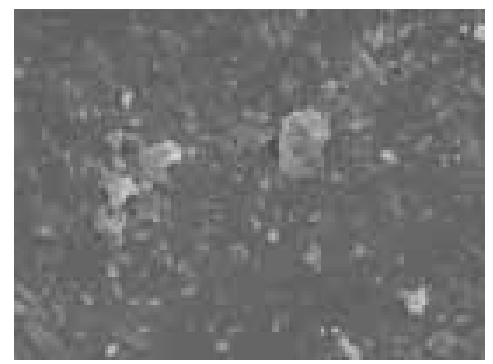

(a)

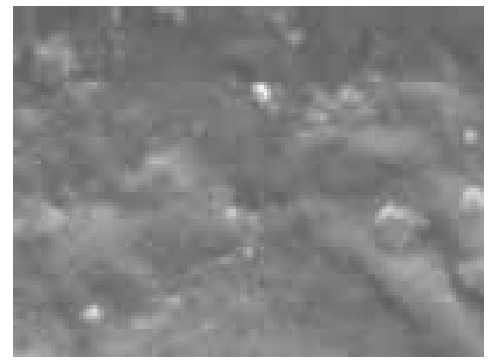

(b)

Figura 5. Fotomicrografias das formulações contendo: (a) PCL com $25 \%$ de amido e (b) PCL com $50 \%$ de amido incorporado com aumento de 10000 vezes.

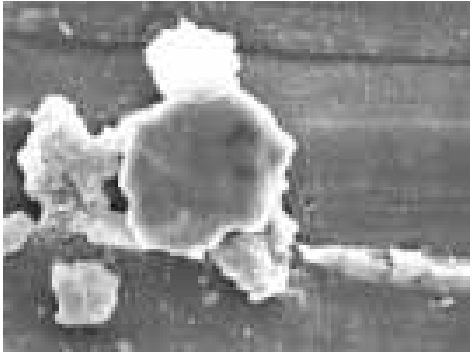

(a)

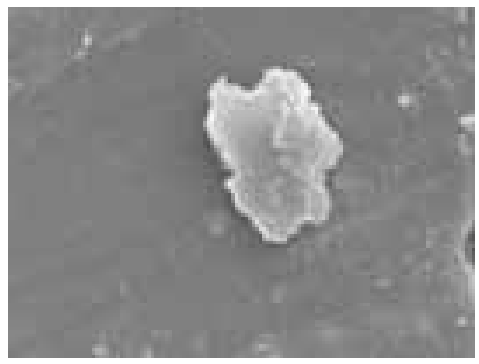

(b)

Figura 6. Fotomicrografias das formulações contendo: (a) PHB com $25 \%$ de amido e (b) PHB com 50\% de amido incorporado com aumento de 10000 vezes.

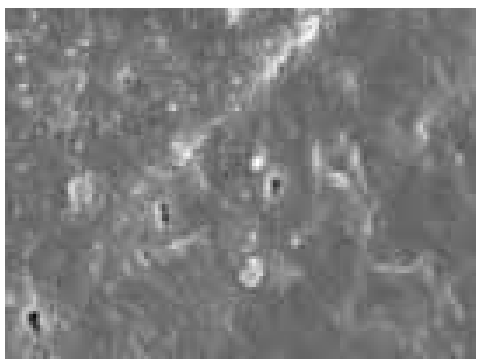

(a)

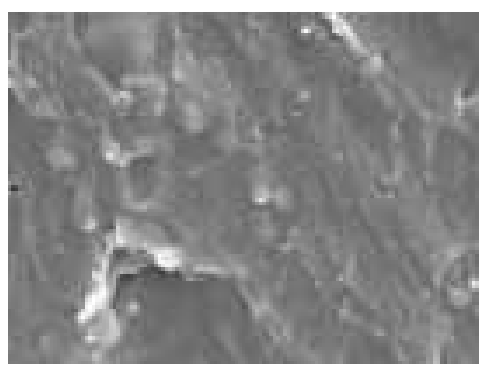

(b)

Figura 7. Fotomicrografias das formulações contendo: (a) PHBV com $25 \%$ de amido e (b) PHBV com 50\% de amido incorporado com aumento de 10000 vezes.

polímero. Para o PHB foi possível visualizar a separação de fase entre este e o amido. No PHBV o comportamento foi similar aos compostos de PCL, no entanto, para as misturas de PHBV foram observados alguns espaços vazios, provavelmente devido a eliminação de gases que estão absorvidos no amido, conforme pode ser constatado na Figura 7. 


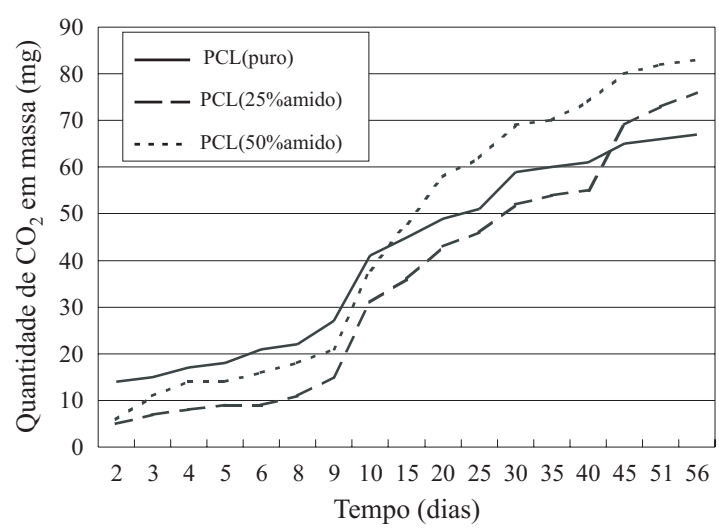

Figura 8. Evolução de $\mathrm{CO}_{2}$ do polímero $\mathrm{PCL}$ e suas blendas quando exposto em lodo ativado.

\section{Liberação de $\mathrm{CO}_{2}$ quando exposto em lodo ativado}

Os resultados da evolução do dióxido de carbono encontrados para os polímeros PCL, PHB, PHBV e suas respectivas misturas estão ilustrados nas Figuras 8,9 e 10 , respectivamente.

Através das Figuras 8, 9 e 10, pode ser observado que a maior liberação de $\mathrm{CO}_{2}$ ocorrida foi com o PHB (Figura 9). No entanto, com a adição de amido não foi constatado um aumento na liberação de $\mathrm{CO}_{2}$ para misturas contendo diferentes teores de material.

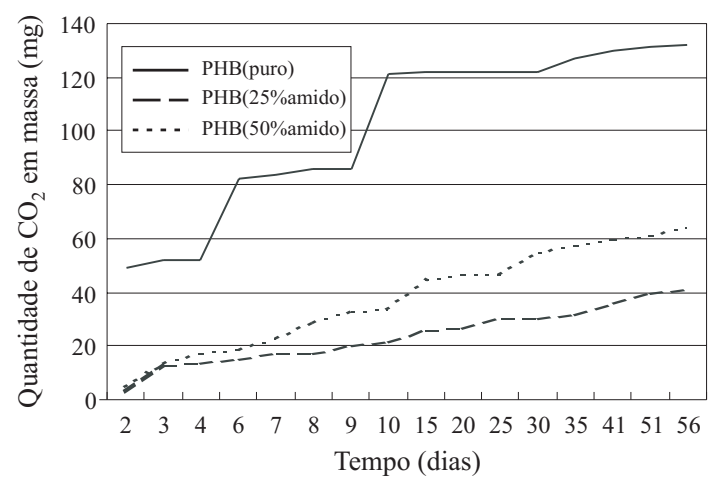

Figura 9. Evolução de $\mathrm{CO}_{2}$ do polímero $\mathrm{PHB}$ e suas misturas quando exposto em lodo ativado.

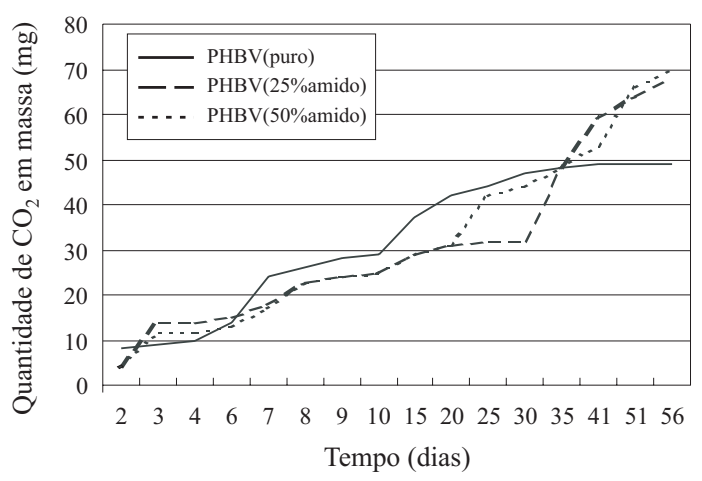

Figura 10. Evolução de $\mathrm{CO}_{2}$ do polímero PHBV e suas misturas quando exposto em lodo ativado.
Para o PCL foi observado que, com o aumento na quantidade de amido na mistura, ocorre uma maior liberação de $\mathrm{CO}_{2}$, com o passar dos dias. $\mathrm{O}$ mesmo comportamento foi constatado para o PHBV. Este comportamento é observado pois as temperaturas de preparação das misturas de PCL foram inferiores a temperatura em que ocorre a gelatinização do amido (85-90 $\left.{ }^{\circ} \mathrm{C}\right)$, ou seja, condição onde ocorre a abertura dos grãos deste material e forma o amido termoplástico. Para o caso das misturas do PHBV, a temperatura de processamento foi superior a de gelatinização do amido e, provavelmente, ocorreu a formação do amido termoplástico, porém, no entanto, a estrutura formada é ainda mais biodegradável que do polímero puro, pois o amido contém cerca de $70 \%$ em massa de amilose, que possui dois importantes grupos funcionais: os grupos $\mathrm{OH}$, suscetíveis de reação de substituição, e a ligação $\mathrm{C}-\mathrm{O}-\mathrm{C}$, que possibilita a ruptura da cadeia e pode sofrer degradação mais facilmente que os polímeros puros ${ }^{[3]}$.

\section{Conclusões}

$\mathrm{Na}$ incorporação do amido nos polímeros puros ocorreu mudança na morfologia e, quanto maior a quantidade de amido incorporado aos polímeros puros, maior foi a redução na sua propriedade mecânica. No entanto, a incorporação de cerca de $25 \%$ em massa de amido, permitiu ainda a obtenção de materiais com valores mínimos de tensão de tração na ruptura de $10 \mathrm{MPa}$. Nos materiais contendo 50\% de PHB com 50\% de amido incorporado, devido a imiscibilidade dos materiais, que pode ser observada com maior clareza na caracterização por microscopia eletrônica de varredura (MEV), ocorreu uma grande redução na propriedade mecânica.

O material que obteve a maior taxa de degradação quando submetido em lodo ativado foi o polímero PHB puro. Quando aumentou-se as dosagens de amido no PCL e no PHBV, observou-se um aumento da taxa de degradação dos mesmos, o que evidencia a viabilidade de obtenção de polímeros biodegradáveis de menor preço.

\section{Agradecimentos}

Os autores agradecem: as empresas Copersucar (Cooperativa de Produtores de Cana, Açúcar e Álcool do Estado de São Paulo Ltda.), Union Carbide Química Ltda. e Corn Products Brasil pelo forneci- 
mento das matérias primas; a Fundação de Amparo à Pesquisa do Estado de São Paulo - FAPESP, pelo incentivo através do PROJETO - PROCESSO $n^{\circ}$ 1999/ 10716-4 e ao CNPq, pela bolsa de iniciação científica fornecida ao aluno Bruno Luiz Marson Franco.

\section{Referências Bibliográficas}

1. Chiellini, E. \& Solaro,R. "Environmentally Degradable Polymers and Plastics. An Overwiew”, in: Anais do International Workshop on Environmentally Degradable and Recyclabe Polymers in Latin America, p. 15-20, Campinas - SP, Nov (1998).

2. Vaydia, U. R., Bhattacharya, M. \& Zhang, D. - Polymer, 36, p.1179, (1995).

3. Huang, J. C.; Shetty A. S. E Wang M. S. - Adv. Polym. Technol., 10, p.23, (1990).

4. Raghavan, D. Polym. Plast. Technol. Eng., $\underline{34}$ (1), p.4163 (1995).
5. Stevens, M. P. - "Polymer Chemistry an Introduction"; Oxford University Press, New York, (1999).

6. Bagley, E. B., Fanta, G. F. E Burr . R. C. Polym. Eng. Sci., 17, p 311-6, (1977).

7. Barham, P.j.; Keller, A.; Otun, E. L. E Holmes, P. A. J. Mat. Sci., 19, p.2781 (1984).

8. Gogolewski, S., Janvanovic, M., Perren, S.m. Dillon, J.g. E Hughes, G. Polym. Degrad. and Stab., 40 , p.313-322, (1993).

9. Informações comerciais obtida da Union Carbide Química Ltda.

10. Norma Astm; American Society for Testing E Materials) designation: D 5209-92. Standard test Method for Determining the Aerobic Biodegradation of Plastics Materials in de Presence of Municipal Sewage; Sludge.p.1-4., (1992).

Recebido: $11 / 12 / 00$

Aprovado: 08/02/01 